\title{
Stents Farmacológicos vs. Stents Não-Farmacológicos no Tratamento de Enxertos de Veia Safena
}

\author{
Carlos A. Collet ${ }^{1}$, J. Ribamar Costa Jr. ${ }^{1,2}$, Amanda G. M. R. Sousa ${ }^{1,2}$, Fausto Feres ${ }^{1}$, Adriana Moreira ${ }^{2}$, \\ Ricardo Costa ${ }^{1,2}$, Rodolfo Staico, ${ }^{1,2}$, Alejandro Almeida1, Dimytri Siqueira', Manuel Cano' ${ }^{2}$, \\ Galo Maldonado ${ }^{1,2}$, Alexandre Abizaid', J. Eduardo Sousa ${ }^{1,2}$
}

\section{RESUMO}

Introdução: Embora os stents farmacológicos tenham reduzido acentuadamente a reestenose e a necessidade de novas revascularizações na maioria dos cenários clínicos e angiográficos, o benefício desses dispositivos no tratamento de lesões em enxertos de veia safena (EVS) ainda precisa ser definido. O objetivo deste estudo foi comparar a incidência de eventos cardíacos adversos maiores (ECAM) entre stents farmacológicos e stents não-farmacológicos em lesões em EVS em pacientes não-selecionados. Métodos: Foram incluídos pacientes tratados em dois hospitais terciários entre maio de 2006 e janeiro de 2009. O objetivo primário foi comparar a incidência de ECAM entre stents farmacológicos e stents não-farmacológicos durante a hospitalização e no seguimento a longo prazo. Resultados: No total, foram incluídos, consecutivamente, 308 pacientes, divididos de acordo com o tipo de stent implantado (209 com stents farmacológicos e 99 com stents não-farmacológicos). A média de idade do grupo foi de 68 anos e a dos EVS, de 10,9 anos. Na fase intra-hospitalar, houve tendência para maior incidência de ECAM no grupo que recebeu stents farmacológicos $(12 \%$ vs. $5,1 \%$; $P=0,06)$. Até 24 meses, a incidência de ECAM foi equivalente nos dois grupos $(17,2 \%$ no grupo stents farmacológicos vs. $18,2 \%$ no grupo stents não-farmacológicos; $P=0,87$ ). Trombose do stent definitiva/provável foi semelhante em ambos os grupos $(2,3 \%$ no grupo stents farmacológicos vs. $2 \%$ no grupo stents não-farmacológicos; $P=0,94)$. Conclusões: Nesta série de pacientes complexos do mundo real, não houve preocupação com a segurança a longo prazo relacionada ao uso de stents farmacológicos no tratamento de lesões em EVS, com incidências similares de morte cardíaca/infarto do miocárdio/trombose do stent em ambos os grupos.

DESCRITORES: Stents. Stents farmacológicos. Veia safena.

\begin{abstract}
Drug-Eluting Stents vs. Bare-Metal Stents in Saphenous Vein Graft Lesions

Background: Although drug-eluting stents have markedly reduced restenosis and the need for repeat lesion revascularization in the vast majority of clinical and angiographic scenarios, the benefit of these devices for the treatment of saphenous-vein graft (SVG) lesions is yet to be defined. The aim of our study was to compare the rate of major adverse clinical events (MACE) between drug-eluting stents and bare-metal stents in SVG lesions in non-selected patients. Methods: Patients treated in two tertiary hospitals between May 2006 and January 2009 were included. The primary objective was to compare the incidence of MACE between drug-eluting stents and bare-metal stents during hospitalization and in the long-term follow-up. Results: Overall, 308 patients were consecutively enrolled and divided according to the type of stent deployed (209 with drug-eluting stents and 99 with bare-metal stents). Mean age was 68 years and SVG mean age was 10.9 years. In the in-hospital phase there was a trend towards higher MACE in the drug-eluting stents group ( $12 \%$ vs. $5.1 \%$; $\mathrm{P}=0.06)$. The incidence of $\mathrm{MACE}$ in up to 24 months was equivalent between groups $(17.2 \%$ for the drug-eluting stents group vs. $18.2 \%$ for the baremetal stents group; $\mathrm{P}=0.87)$. Definite/probable stent thrombosis was similar in both groups $(2.3 \%$ for the drug-eluting stents group vs. $2 \%$ for bare-metal stents group; $\mathrm{P}=0.94$ ). Conclusions: In this real world series of complex patients there was no long-term safety concerns related to the use of drug-eluting stents in the treatment of SVG lesions, with similar rates of cardiac death/myocardial infarction/stent thrombosis in both groups.
\end{abstract}

KEY-WORDS: Stents. Drug-eluting stents. Saphenous vein.

\footnotetext{
Instituto Dante Pazzanese de Cardiologia - São Paulo, SP, Brasil. 2 Hospital do Coração - Associação Sanatório Sírio - São Paulo, SP, Brasil.

Correspondência: J. Eduardo Sousa. Hospital do Coração - Associação do Sanatório Sírio - Rua Desembargador Eliseu Guilherme, 147 Paraíso - São Paulo, SP, Brasil - CEP 04004-030

E-mail: jesousa@uol.com.br

Recebido em: 28/2/2011 • Aceito em: 13/5/2011
} 
0 advento de stents farmacológicos há cerca de uma década mudou o resultado das intervenções coronárias percutâneas (ICPs) em artérias coronárias, reduzindo acentuadamente a incidência de reestenose nos mais variados e complexos cenários clínicos e angiográficos, quando comparados com os stents não-farmacológicos. ${ }^{1}$ Meta-análises recentes não apenas demonstraram a clara superioridade dessa nova tecnologia quanto à necessidade de repetir a revascularização das lesões, como também mostraram perfil de segurança semelhante ao dos stents não-farmacológicos, tanto em ensaios clínicos controlados como em populações do mundo real. ${ }^{2-5}$ Entretanto, ainda há controvérsias quanto ao uso de stents farmacológicos no tratamento de lesões em enxertos de veia safena (EVS). A escassez de dados de estudos randomizados e a falta de grandes registros com seguimento prolongado (> 1 ano) são responsáveis pela incerteza em relação à indicação desses dispositivos no tratamento de lesões em EVS. ${ }^{6,7}$

O objetivo do presente estudo foi comparar a segurança e a eficácia a longo prazo ( $\geq 1$ ano) entre stents farmacológicos e stents não-farmacológicos em lesões em EVS em pacientes não-selecionados da prática clínica diária.

\section{MÉTODOS}

Este registro incluiu pacientes consecutivos com lesões em EVS tratados em dois centros (um hospital público e um privado) pela mesma equipe de investigadores entre maio de 2006 e janeiro de 2009. Foram excluídos os pacientes tratados na vigência de infarto do miocárdio (IM) agudo e aqueles com reestenose intrastent. Como o estudo foi baseado em desfechos clínicos, os pacientes que receberam ambos os tipos de stent (stent farmacológico e stent não-farmacológico) também foram excluídos desta análise.

O estudo foi aprovado pelo Comitê de Ética Institucional. Todos os pacientes assinaram o Termo de Consentimento Informado antes do procedimento. As instituições e os participantes não receberam qualquer espécie de apoio financeiro para desenvolver esta pesquisa.

\section{Procedimento de implante dos stents}

Todas as intervenções foram realizadas de acordo com as diretrizes atuais e a estratégia dos procedimentos ficou a critério dos operadores. Os pacientes tratados no hospital público receberam stents não-farmacológicos, enquanto os stents farmacológicos foram a opção padrão no hospital privado.

O tipo de stent implantado, a estratégia de pré e/ou pós-dilatação e o uso de dispositivos de proteção distal também ficaram a critério do investigador. Tratamento antiplaquetário, que incluiu aspirina (dose de ataque de $200 \mathrm{mg}$ a $325 \mathrm{mg}$ ) e tienopiridínicos (ticlopidina $250 \mathrm{mg}$ a cada 12 horas ou clopidogrel $300 \mathrm{mg}$ ), foi iniciado pelo menos 24 horas antes dos procedimentos eletivos; nos casos de urgência, dose de ataque de $600 \mathrm{mg}$ de clopidogrel foi administrada imediatamente antes da intervenção. Após o procedimento, a aspirina foi continuada indefinidamente e o tienopiridínico foi mantido por apenas um mês após o implante de stents não-farmacológicos e por 12 meses após o implante de stents farmacológicos. Durante o procedimento, foi administrada heparina por via intravenosa (70 Ul/kg a $100 \mathrm{UI} / \mathrm{kg}$ ), após a inserção da bainha, para manter o tempo de coagulação ativada $>250$ segundos. O uso adicional de medicamentos durante o procedimento, inclusive inibidores da glicoproteína Ilb/IIla, foi deixado a critério do investigador. Foi obtido eletrocardiograma de 12 derivações antes, imediatamente após e 24 horas depois do procedimento. Análise laboratorial incluiu creatina quinase $(C K)$ e sua fração $M B$ (CK-MB) antes do procedimento $(<24$ horas) e 12-18 horas após o tratamento.

\section{Análise angiográfica}

Após a administração intracoronária de nitrato (100$200 \mu \mathrm{g})$, foi obtida angiografia coronária seriada antes e após o procedimento. Foi realizada análise coronária quantitativa (ACQ) off-line usando o sistema semiautomático de análise computadorizada de detecção de bordas CMS-GFT ${ }^{\mathrm{TM}}$ versão 5.1 (Medis, Leiden, Holanda). O diâmetro mínimo da luz e o diâmetro de referência, obtido a partir da média dos segmentos de $5 \mathrm{~mm}$ proximais e distais à lesão-alvo (ou lesões-alvo), foram usados para calcular o diâmetro da estenose: $\mathrm{DE}=(1-\mathrm{DML} / \mathrm{DR})$ x 100, em que DE = diâmetro da estenose, $\mathrm{DML}=$ diâmetro mínimo da luz, e DR = diâmetro de referência. O ganho agudo foi obtido a partir da diferença do diâmetro mínimo da luz pré e pós-procedimento.

Todas as análises foram avaliadas por um mesmo operador, que permaneceu cego em relação aos dados do procedimento.

\section{Desfechos, definições e seguimento clínico}

O objetivo primário do estudo foi a ocorrência de eventos cardíacos adversos maiores (ECAM) e de trombose do stent em acompanhamento clínico $\geq 1$ ano.

ECAM foi definido como morte cardíaca, IM nãofatal ou revascularização da lesão-alvo (RLA). RLA foi realizada apenas na presença de sintomas e/ou sinais de isquemia. Todas as mortes foram consideradas cardíacas, a não ser que uma origem não-cardíaca pudesse ser claramente estabelecida por estudo clínico e/ou patológico.

O diagnóstico de IM foi baseado no desenvolvimento de novas ondas $Q$ patológicas em $\geq 2$ derivações eletrocardiográficas contíguas e/ou na elevação da isoenzima CK-MB > 3 vezes o limite superior normal após o procedimento, durante a hospitalização índice, ou $>2$ vezes o limite superior normal após a alta hospitalar. 
Trombose do stent foi classificada como definitiva, provável e possível, de acordo com as definições propostas pelo Academic Research Consortium (ARC), e estratificada em aguda ( $<24$ horas), subaguda (24 horas a 30 dias), tardia (31 dias a 12 meses) e muito tardia (> 12 meses). ${ }^{8}$

Sucesso angiográfico foi definido como obtenção de estenose residual $<20 \%$ por ACQ no segmento tratado após o implante do stent farmacológico. Sucesso do procedimento foi definido como sucesso angiográfico e ausência de ECAM durante a hospitalização. No período de inclusão, foram obtidos, de cada paciente, dados demográficos detalhados e informações clínicas, angiográficas e relativas ao procedimento, inclusive complicações. O seguimento clínico foi baseado em visitas ambulatoriais ou em contatos telefônicos, e registrado em fichas clínicas. No momento do seguimento, foram coletados dados referentes a estado clínico atual, medicação concomitante (com ênfase especial no regime antiplaquetário), e ocorrência, nesse ínterim, de eventos adversos pré-definidos. Os dados pessoais dos pacientes foram codificados, para evitar a identificação dos sujeitos participantes do estudo.

O seguimento angiográfico rotineiro não fez parte do protocolo do estudo. Dessa maneira, todas as reintervenções foram indicadas por critérios clínicos.

\section{Análise estatística}

As variáveis qualitativas foram expressas como porcentagens e as variáveis quantitativas, como média \pm desvio padrão. A comparação das variáveis numéricas foi realizada por meio do teste de Mann-Whitney ou do teste de Pearson, quando apropriado. O teste do qui-quadrado ou o teste exato de Fischer foram usados para comparar variáveis qualitativas. A sobrevida livre de ECAM, morte cardíaca, IM e RLA foi estimada por meio do método de Kaplan-Meier e as diferenças entre as curvas foram comparadas com o teste log-rank. Adicionalmente, foi realizada uma análise excluindo os eventos que ocorreram dentro de 30 dias após o procedimento, portanto não relacionados ao tipo de stent implantado. O modelo de regressão de Cox para riscos proporcionais foi usado para identificar os preditores independentes de ECAM. As diferenças foram consideradas estatisticamente significantes com valor de $\mathrm{P}<0,05$, e todos os valores de $\mathrm{P}$ relatados foram bicaudais. Os dados foram analisados por meio do software SPSS versão 12.0 (SPSS Inc., Chicago, Estados Unidos).

\section{RESULTADOS}

\section{Características basais e do procedimento}

Foram incluídos 308 pacientes, divididos de acordo com o tipo de stent implantado (209 pacientes tratados com stents farmacológicos e 99 com stents não-farmacológicos).

A média de idade do grupo foi de 68 anos e dos EVS, de 10,9 anos, sem diferença significativa entre os dois grupos. Houve maior frequência de mulheres $(23,2 \%$ vs.

TABELA 1

Características clínicas basais

\begin{tabular}{lccc}
\hline & Grupo SF $(\mathbf{n}=\mathbf{2 0 9})$ & Grupo SNF $(\mathbf{n}=\mathbf{9 9})$ & P \\
\hline Idade, anos & $68,1 \pm 9,9$ & $68 \pm 10$ & 0,87 \\
Sexo masculino, n (\%) & $182(87,1)$ & $76(76,8)$ & 0,02 \\
Diabetes mellitus, n (\%) & $66(31,6)$ & $50(50,5)$ & 0,001 \\
Hipercolesterolemia, n (\%) & $153(73,2)$ & $73(73,7)$ & 0,92 \\
Hipertensão arterial, n (\%) & $170(81,3)$ & $10(10,1)$ & 0,09 \\
Tabagismo, n (\%) & $53(25,4)$ & $58(58,6)$ & 0,002 \\
IM anterior, n (\%) & $68(32,5)$ & $68,2 \pm 21$ & 0,001 \\
Clearance de creatinina, ml/min & $67,3 \pm 24$ & $11,4 \pm 5,6$ & 0,35 \\
Cirurgia de revascularização, anos & $10,6 \pm 4,8$ & \\
Apresentação clínica, n (\%) & & $69(69,7)$ & 0,32 \\
Angina estável/isquemia silenciosa & $137(65,6)$ & $30(30)$ & \\
IAMSST & $72(34,4)$ & $39(39,4)$ & 0,14 \\
Fração de ejeção, n (\%) & & $38(38,4)$ & $3(3)$ \\
$>55 \%$ & $58(27,8)$ & $92(44)$ &
\end{tabular}

IAMSST = infarto agudo do miocárdio sem supradesnivelamento do segmento ST; IM = infarto do miocárdio; $\mathrm{n}$ = número de pacientes; SF = stent farmacológico; SNF = stent não-farmacológico. 
12,9\%; $P=0,02)$, de diabéticos $(50,5 \%$ vs. 31,6\%; $P=0,001)$ e de IM prévio $(58,6 \%$ vs. $32,5 \% ; P<0,001)$ entre os pacientes que receberam stents não-farmacológicos. Ao contrário, pacientes fumantes $(25,4 \%$ vs. $10,1 \% ; P=0,002$ ) foram mais prevalentes entre os que receberam stents farmacológicos. Outras características clínicas basais não diferiram entre os grupos (Tabela 1).

As características angiográficas e do procedimento estão apresentadas na Tabela 2. O território mais frequentemente tratado foi o suprido pelas artérias circunflexa/obtusa marginal, seguidas pela artéria coronária direita e pela artéria descendente anterior, sem diferença entre os grupos $(P=0,25)$. Comparativamente aos pacientes tratados com stents farmacológicos, os pacientes tratados com stents não-farmacológicos re- ceberam mais frequentemente inibidores da glicoproteína IIb/IIla $(2,4 \%$ vs. $13,3 \% ; \mathrm{P}<0,001)$ e usaram mais dispositivos de proteção distal (5,9\% vs. 35,7\%; $\mathrm{P}<0,001)$. Em comparação com o grupo tratado com stents não-farmacológicos, os EVS foram menos frequentemente pré-dilatados (27,9\% vs. 42,9\%; P = 0,009) e pós-dilatados $(31,3 \%$ vs. 54,1\%; $\mathrm{P}<0,001)$ nos pacientes do grupo tratado com stents farmacológicos, e em mais da metade desses pacientes foi implantado stent eluidor de sirolimus (Cypher $\left.{ }^{\mathrm{TM}}\right)$. Diâmetro vascular de referência pré-procedimento, comprimento da lesão e diâmetro da estenose foram semelhantes em ambos os grupos. A ACQ pós-procedimento revelou ganho imediato e estenose residual semelhantes. Sucesso angiográfico foi obtido em $\geq 97 \%$ dos casos em ambos os grupos $(P=0,5)$.

TABELA 2

Características angiográficas e do procedimento

\begin{tabular}{|c|c|c|c|}
\hline & Grupo SF (n = 209) & Grupo SNF (n = 99) & $\mathbf{P}$ \\
\hline Lesões, n & 268 & 106 & NA \\
\hline Território tratado, n (\%) & & & 0,25 \\
\hline Descendente anterior/diagonal & $65(31,3)$ & $22(22,2)$ & \\
\hline Circunflexa/obtusa marginal & $73(35,1)$ & $41(41,4)$ & \\
\hline Coronária direita & $70(33,7)$ & $36(36,4)$ & \\
\hline Localização da lesão, n (\%) & & & 0,08 \\
\hline Ostial & $30(14,4)$ & $25(25,5)$ & \\
\hline Corpo/anastomose distal & $238(85,6)$ & $81(74,5)$ & \\
\hline Inibidor da glicoproteína IIb/IIla, n (\%) & $5(2,4)$ & $13(13,3)$ & $<0,001$ \\
\hline Dispositivo de proteção distal, n (\%) & $11(5,9)$ & $35(35,7)$ & $<0,001$ \\
\hline Pré-dilatação, n (\%) & $58(27,9)$ & $42(42,9)$ & 0,009 \\
\hline Pós-dilatação, n (\%) & $65(31,3)$ & $53(54,1)$ & $<0,001$ \\
\hline Comprimento total do stent, $\mathrm{mm}$ & $20,6 \pm 8$ & $23,1 \pm 13,9$ & 0,98 \\
\hline Pressão máxima de insuflação, atm & $15,6 \pm 3$ & $15,4 \pm 3,8$ & 0,73 \\
\hline Stents por lesão, número & $1,08 \pm 0,2$ & $1,16 \pm 0,4$ & 0,06 \\
\hline Tipo de SF implantado, n (\%) & & & NA \\
\hline Cypher $^{\mathrm{TM}}$ & $171(60)$ & 0 & \\
\hline Taxus $^{\mathrm{TM}}$ & $54(18,9)$ & 0 & \\
\hline Endeavor $^{\mathrm{TM}}$ & $34(11,9)$ & 0 & \\
\hline Resolute $^{\mathrm{TM}}$ & $6(2)$ & 0 & \\
\hline Biomatrix $^{\mathrm{TM}}$ & $3(1)$ & 0 & \\
\hline Promus $^{\mathrm{TM}}$ & $7(2,5)$ & 0 & \\
\hline Xience $V^{T M}$ & $12(4)$ & 0 & \\
\hline Diâmetro de referência, mm & $3,2 \pm 0,6$ & $3,2 \pm 0,8$ & $>0,99$ \\
\hline Comprimento da lesão, mm & $15,5 \pm 7,5$ & $14,8 \pm 8,65$ & 0,75 \\
\hline Diâmetro mínimo da luz, mm & $1,04 \pm 0,4$ & $1,23 \pm 0,5$ & 0,43 \\
\hline Diâmetro da estenose, \% & $67,6 \pm 9,9$ & $60,8 \pm 15$ & 0,14 \\
\hline Estenose residual, \% & $5,1 \pm 3,4$ & $9,1 \pm 4,4$ & 0,1 \\
\hline Ganho imediato, mm & $2 \pm 0,6$ & $1,72 \pm 0,7$ & 0,53 \\
\hline Sucesso angiográfico, \% & 98 & 97 & 0,5 \\
\hline
\end{tabular}

$\mathrm{n}$ = número de pacientes; NA = não aplicável; SF = stent farmacológico; SNF = stent não-farmacológico. 


\section{Resultados clínicos}

Seguimento clínico foi obtido em todos os pacientes de ambos os grupos. Ocorreram, no total, 54 eventos durante o seguimento (mediana de 23 meses, intervalo interquartil de 19,3-24 meses) (Tabela 3). Houve tendência a maior número de ECAM intra-hospitalares no grupo que recebeu stents farmacológicos (12\% vs. $5,1 \% ; P=0,06)$, por causa da maior incidência de IM sem onda $Q(12 \%$ vs. 5,1\%; $\mathrm{P}=0,06)$. Entretanto, o número de ECAM combinados até 24 meses foi equivalente entre os grupos $(17,2 \%$ para o grupo de stents farmacológicos vs. 18,2\% para o grupo de stents nãofarmacológicos; $P=0,87$ ) (Figura 1, painel esquerdo), com ocorrência duas vezes mais alta e não-significativa de RLA entre os pacientes com stents não-farmacológicos (3,3\% vs. 6,2\%; P = 0,24) (Figura 2). Mortes cardíacas e IM foram semelhantes entre os grupos no seguimento a longo prazo. Por causa de uma diferença inicial de ECAM intra-hospitalares decorrente de IM periprocedimento (25 no grupo de stents farmacológicos vs. 5 no grupo de stents não-farmacológicos; $\mathrm{P}=0,06)$, foi realizada uma análise excluindo os primeiros 30 dias de seguimento (Figura 1, painel direito);

TABELA 3

Eventos clínicos intra-hospitalares e tardios

\begin{tabular}{lccc}
\hline & Grupo SF $(\mathbf{n}=\mathbf{2 0 9})$ & Grupo SNF $(\mathbf{n}=\mathbf{9 9})$ & P \\
\hline Eventos intra-hospitalares, n (\%) & $25(12)$ & $5(5,1)$ & 0,06 \\
$\quad$ Morte & 0 & 0 & NA \\
Infarto do miocárdio & $25(12)$ & $5(5,1)$ & 0,06 \\
RLA & 0 & 0 & NA \\
Eventos tardios, n (\%) & $36(17,2)$ & $18(18,2)$ & 0,87 \\
ECAM & $7(3,3)$ & $1(1)$ & 0,25 \\
Morte & $28(13,4)$ & $14(14,4)$ & 0,85 \\
Infarto do miocárdio & $7(3,3)$ & $6(6,2)$ & 0,24 \\
RLA & &
\end{tabular}

ECAM = eventos cardíacos adversos maiores; $\mathrm{n}$ = número de pacientes; NA = não aplicável; RLA = revascularização da lesão-alvo; SF = stent farmacológico; SNF = stent não-farmacológico.

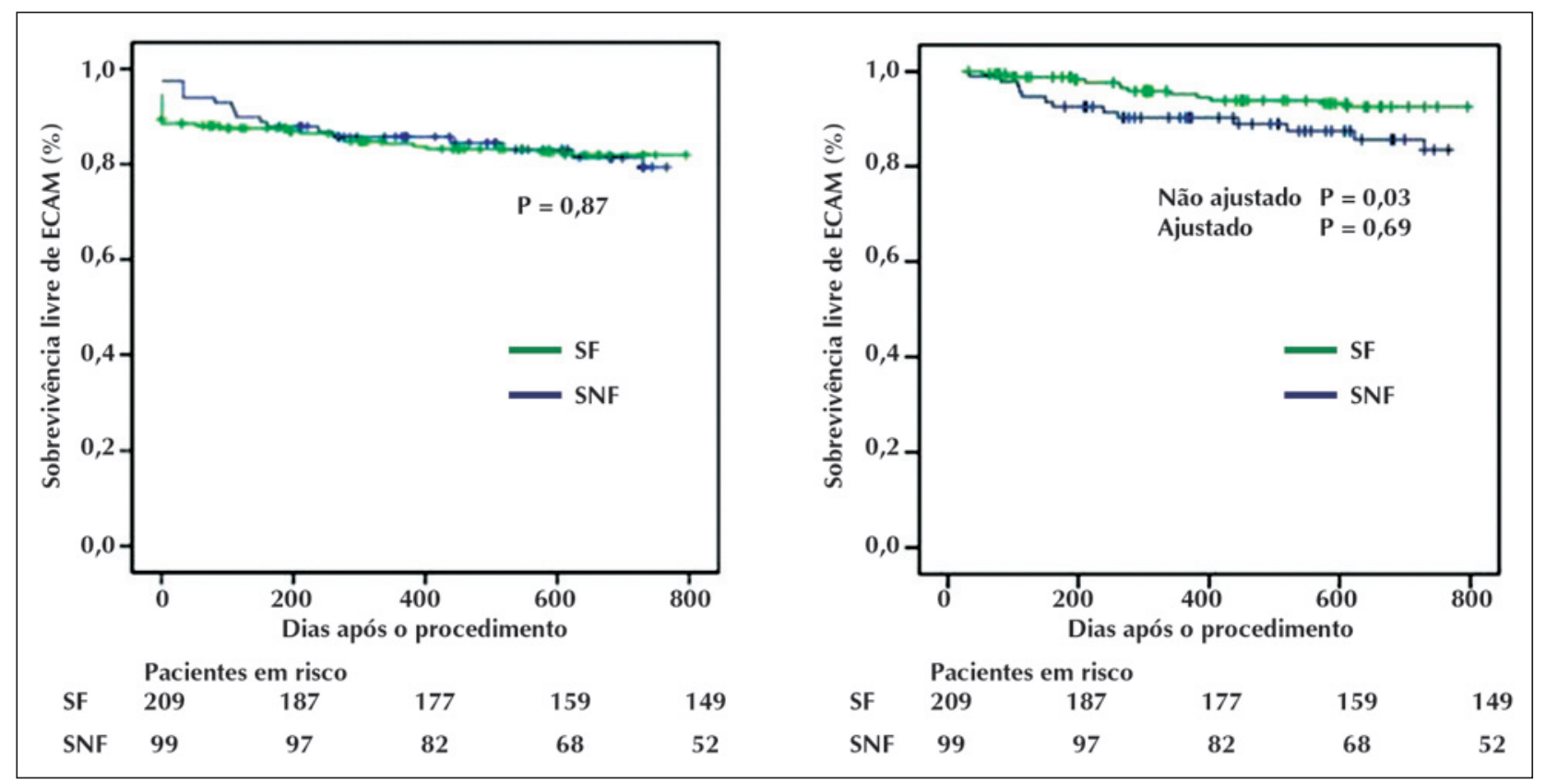

Figura 1 - Painel à esquerda: curvas de sobrevida de Kaplan-Meier para ausência de eventos cardíacos adversos maiores. Painel à direita: curvas de sobrevida de Kaplan-Meier para ausência de eventos cardíacos adversos maiores, começando aos 30 dias do procedimento basal. ECAM = eventos cardíacos adversos maiores; SF = stent farmacológico; SNF = stent não-farmacológico. 


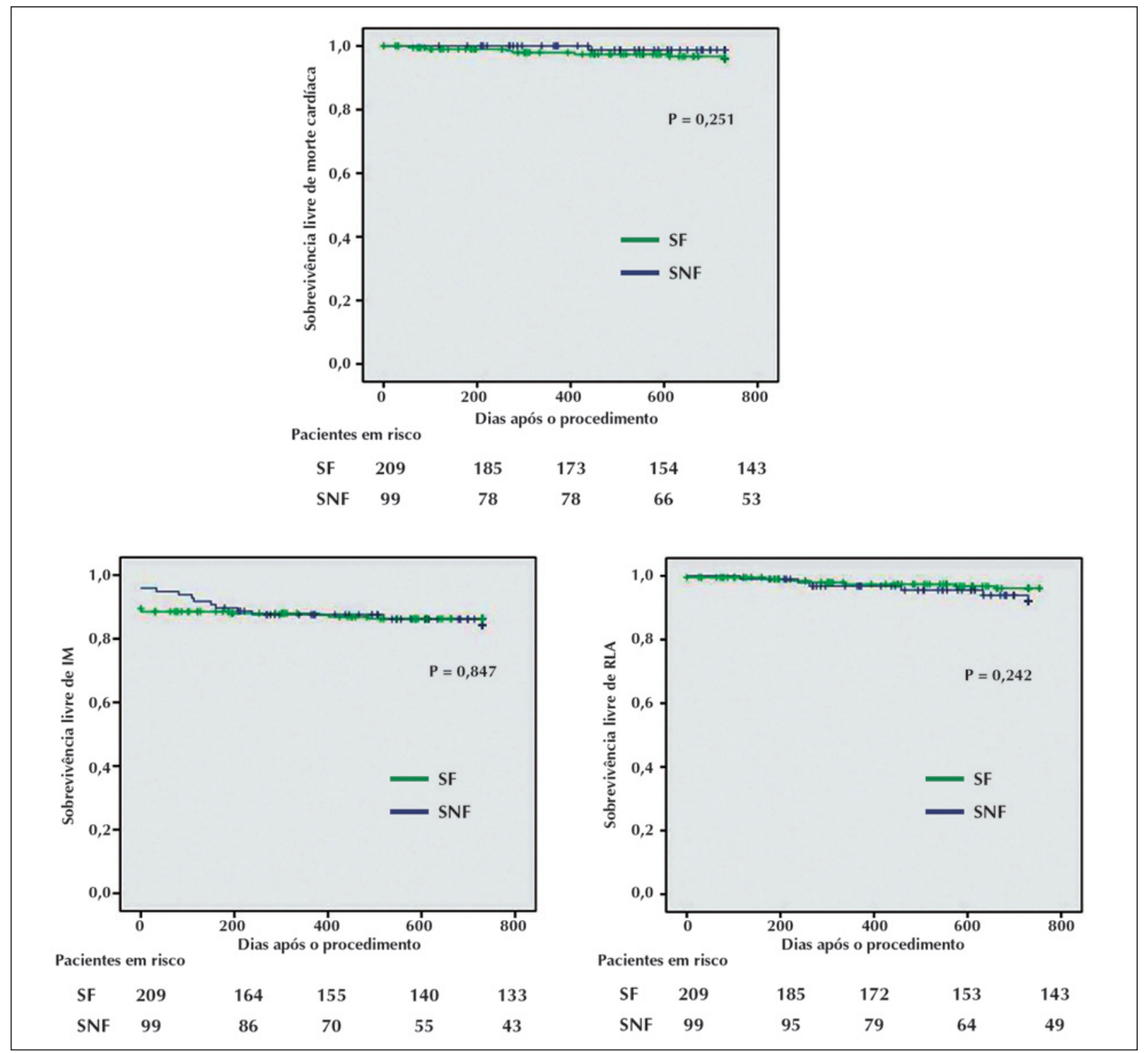

Figura 2 - Curvas de sobrevida de Kaplan-Meier para ausência de morte cardíaca (painel superior), infarto do miocárdio (painel inferior à esquerda) e revascularização da lesão-alvo (painel inferior à direita). IM = infarto do miocárdio; RLA = revascularização da lesão-alvo; SF = stent farmacológico; SNF = stent não-farmacológico.

no modelo não ajustado, foi encontrada diferença significativa favorecendo o grupo de stents farmacológicos em relação à ocorrência de ECAM, que foi perdida após o ajuste para os outros fatores de confusão.

A Tabela 4 apresenta a classificação de todos os casos de trombose do stent, de acordo com o ARC, que apontou não haver diferença significativa entre os grupos. A Figura 3 mostra a incidência de trombose do stent definitiva/provável em ambos os grupos, até dois anos.

Pela análise multivariada, hipercolesterolemia [razão de chance (RC) de 2,8, intervalo de confiança de
95\% (IC 95\%) 1,2-6,9; P = 0,017], função ventricular esquerda $<30 \%$ (RC 3,9, IC 95\% 1,3-11,7; P = 0,015) e comprimento do stent (RC 1,02, IC 95\% 1-1,05; $\mathrm{P}=0,011)$ foram os únicos preditores independentes de ECAM.

\section{DISCUSSÃO}

O principal achado do presente estudo foi a equivalência quanto a segurança e eficácia entre stents farmacológicos e stents não-farmacológicos no tratamento de lesões em EVS sem preocupação específica em relação a morte/IM/trombose do stent no seguimento de até dois anos. 
TABELA 4

Classificação temporal dos casos de trombose de stent

\begin{tabular}{lccc}
\hline & Grupo SF $(\mathbf{n}=\mathbf{2 0 9})$ & Grupo SNF $(\mathbf{n}=\mathbf{9 9})$ & $\mathbf{P}$ \\
\hline Trombose de stent definitiva, $\mathrm{n}(\%)$ & $5(2,4)$ & 0 & 0,18 \\
Precoce (0-30 dias) & 0 & 0 & \\
Tardia (31-360 dias) & $2(1)$ & 0 & \\
Muito tardia (> 360 dias) & $3(1,4)$ & 0 & 0,10 \\
Trombose de stent provável, $\mathrm{n}(\%)$ & 0 & $2(2)$ & \\
Precoce (0-30 dias) & 0 & $1(1)$ & \\
Tardia (31-360 dias) & 0 & $1(1)$ & \\
Muito tardia (> 360 dias) & 0 & & \\
\hline
\end{tabular}

$\mathrm{n}=$ número de pacientes; $\mathrm{SF}=$ stent farmacológico; $\mathrm{SNF}=$ stent não-farmacológico.

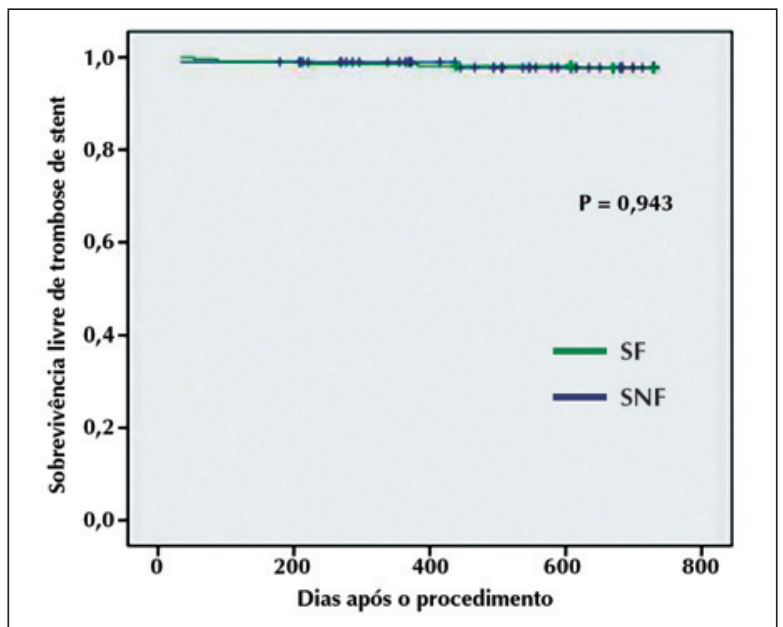

Figura 3 - Curvas de sobrevida de Kaplan-Meier para ausência de trombose de stent definitiva/provável. SF = stent farmacológico; SNF = stent não-farmacológico.

O primeiro estudo randomizado que comparou esses dois tipos de stent em lesões em EVS foi o Reduction of Restenosis in Saphenous Vein Grafts with Cypher ${ }^{T M}$ Sirolimus-Eluting Stent (RRISC). No total, 75 pacientes (96 lesões em EVS) foram randomizados para receber Cypher ${ }^{\mathrm{TM}}$ ou stent não-farmacológico, tendo sido submetidos a avaliação invasiva seriada por meio de ACQ e ultrassom intracoronário (USIC) no momento basal e após seis meses de seguimento. O uso do stent farmacológico foi associado a 37\% de redução do risco de reestenose binária $(11,3 \%$ vs. 30,6\%; $\mathrm{P}<0,024)$, com taxas semelhantes de morte e IM no seguimento a curto prazo. ${ }^{6}$ Análise por USIC também confirmou a acentuada superioridade do stent farmacológico na redução da formação de neoíntima intrastent (1,3\% vs. 24,5\%; $\mathrm{P}<0,001) .{ }^{9}$ Esses mesmos pacientes foram seguidos a longo prazo (mediana de 32 meses) e, surpreendentemente, nesse estudo (estudo DELAYED RRISC), a taxa de morte foi acentuadamente maior entre os tratados com stent farmacológico. Da mesma forma, o benefí- cio inicial de redução de RLA observada no grupo com stent farmacológico não se manteve aos três anos. ${ }^{10}$

Posteriormente, o estudo Stenting of Saphenous Vein Grafts (SOS) comparou 80 pacientes com 112 lesões em EVS tratadas randomicamente com stent nãofarmacológico ou com o stent farmacológico Taxus ${ }^{\mathrm{TM}}$. No seguimento de 12 meses, os pacientes que receberam stent farmacológico apresentaram significativamente menos reestenose binária ( $9 \%$ vs. 51\%; $\mathrm{P}<0,001)$, com taxas semelhantes de morte/IM e de oclusão do enxerto. ${ }^{7}$

Depois desses estudos randomizados iniciais realizados em pequenos grupos, foram apresentadas muitas séries de populações do mundo real com lesões em EVS. ${ }^{11-20}$ Recentemente foram publicadas duas metaanálises de ensaios randomizados e estudos observacionais, abrangendo mais de 5 mil pacientes. Meir et al. ${ }^{21}$ relataram taxas semelhantes de morte, IM e RLA em pacientes tratados com stents farmacológicos e stents não-farmacológicos em ensaios randomizados. Por outro lado, a análise de estudos observacionais apontou redução significativa de morte, IM e RLA entre os pacientes tratados com stents farmacológicos. Na meta-análise conduzida por Sanchez-Recalde et al.22, os pacientes que receberam stents farmacológicos apresentaram taxas significativamente menores de RLA e morte.

Ao se analisar esses dados, pode-se perguntar por que razão há tamanha disparidade entre esses resultados, especialmente quando são comparados dados de estudos randomizados com os de estudos observacionais. Parte dessas diferenças pode ser atribuída ao perfil clínico basal dos pacientes tratados com stents farmacológicos e stents não-farmacológicos e a tendências na seleção dos pacientes que receberam um tipo ou outro de stent em algumas séries do mundo real. Ao contrário, até o momento não há, nesse cenário, qualquer estudo clínico randomizado com poder para avaliar desfechos clínicos. Nesse contexto, os pacientes incluídos neste estudo apresentam perfil de risco 
e características angiográficas semelhantes aos dos pacientes incluídos em ensaios randomizados, o que pode ajudar a explicar os resultados deste estudo.

Tão importante quanto determinar o melhor tipo de stent para tratar lesões em EVS é entender que a progressão das lesões ateroscleróticas é fundamentalmente diferente nas artérias coronárias nativas e nos EVS. Nos últimos, a progressão das lesões parece ser mais rápida, mais difusa e mais grave, o que pode explicar por que os benefícios dos novos dispositivos nesse cenário parecem diminuir com o passar dos meses, com altas taxas de oclusão do enxerto documentadas em estudos com seguimento invasivo. ${ }^{5,6,13}$ Recentemente publicado, o ensaio Moderate Vein Graft Lesion Stenting with the Taxus Stent and Intravascular Ultrasound (VELETI) revelou informações sobre a progressão da doença em EVS no local de placas moderadas, com $22 \%$ de oclusão dos EVS ou lesões limitantes do fluxo aos 12 meses de acompanhamento em pacientes tratados clinicamente. ${ }^{23}$

Por todas essas razões, é razoável afirmar que pacientes com lesões em EVS representam uma das populações mais desafiadoras a serem tratadas dentro do espectro das doenças das artérias coronárias e que o tipo de stent usado dificilmente irá modificar o resultado naturalmente desfavorável nesses indivíduos.

\section{CONCLUSÕES}

Nesta série de pacientes complexos do mundo real, não houve preocupação com a segurança a longo prazo relacionada ao uso de stents farmacológicos no tratamento de lesões em EVS, com incidências similares de morte cardíaca/infarto do miocárdio/trombose do stent em ambos os grupos. Entretanto, o benefício desse novo dispositivo, em termos de eficácia, pode ter sido diminuído pela progressão aterosclerótica mais rápida encontrada nesses EVS.

\section{CONFLITO DE INTERESSES}

Os autores declaram não haver conflito de interesses relacionado a este manuscrito.

\section{REFERÊNCIAS}

1. Morice MC, Serruys PW, Sousa JE, Fajadet J, Ban Hayashi E, Perin $M$, et al. RAVEL Study Group. Randomized Study with the Sirolimus-Coated Bx Velocity Balloon-Expandable Stent in the Treatment of Patients with de Novo Native Coronary Artery Lesions. A randomized comparison of a sirolimuseluting stent with a standard stent for coronary revascularization. N Engl J Med. 2002;346(23):1773-80.

2. Kirtane AJ, Gupta A, lyengar S, Moses JW, Leon MB, Applegate $R$, et al. Safety and efficacy of drug-eluting and bare metal stents: comprehensive meta-analysis of randomized trials and observational studies. Circulation. 2009;119(25):3198-206.

3. Stettler C, Allemann S, Wandel S, Kastrati A, Morice MC, Schömig $A$, et al. Drug eluting and bare metal stents in people with and without diabetes: collaborative network meta-analysis. BMJ. 2008;337:a1331.
4. Piscione F, Piccolo R, Cassese S, Galasso G, De Rosa R, $D^{\prime}$ Andrea $C$, et al. Effect of drug-eluting stents in patients with acute ST-segment elevation myocardial infarction undergoing percutaneous coronary intervention: a meta-analysis of randomised trials and an adjusted indirect comparison. Eurolntervention. 2010;5(7):853-60.

5. Saeed B, Kandzari DE, Agostoni P, Lombardi WL, Rangan $\mathrm{BV}$, Banerjee S, et al. Use of drug-eluting stents for chronic total occlusions: a systematic review and meta-analysis. Catheter Cardiovasc Interv. 2011;77(3):315-32.

6. Vermeersch $\mathrm{P}$, Agostoni $\mathrm{P}$, Verheye $\mathrm{S}$, Van den Heuvel $\mathrm{P}$, Convens C, Bruining N, et al. Randomized double-blind comparison of sirolimus-eluting stent versus bare-metal stent implantation in diseased saphenous vein grafts: sixmonth angiographic, intravascular ultrasound, and clinical follow-up of the RRISC Trial. J Am Coll Cardiol. 2006;48(12): 2423-31.

7. Brilakis ES, Lichtenwalter C, Lemos JA, Roesle M, Obel O, Haagen D, et al. A randomized controlled trial of a paclitaxeleluting stent versus a similar bare-metal stent in saphenous vein graft lesions: the SOS (Stenting of Saphenous Vein Grafts) trial. J Am Coll Cardiol. 2009;53(11):919-28.

8. Cutlip DE, Windecker S, Mehran R, Boam A, Cohen DJ, van Es GA, et al.; Academic Research Consortium. Clinical end points in coronary stent trials: a case for standardized definitions. Circulation. 2007;115(17):2344-51.

9. Agostoni $\mathrm{P}$, Vermeersch $\mathrm{P}$, Semeraro $\mathrm{O}$, Verheye $\mathrm{S}$, Van Langenhove G, Van den Heuvel P, et al. Intravascular ultrasound comparison of sirolimus-eluting stent versus bare metal stent implantation in diseased saphenous vein grafts (from the RRISC [Reduction of Restenosis In Saphenous Vein Grafts With Cypher Sirolimus-Eluting Stent] trial). Am J Cardiol. 2007; 100(1):52-8.

10. Vermeersch $P$, Agostoni $P$, Verheye $S$, Van den Heuvel $P$, Convens C, Van den Branden F, et al.; DELAYED RRISC (Death and Events at Long-term follow-up AnalYsis: Extended Duration of the Reduction of Restenosis In Saphenous vein grafts with Cypher stent) Investigators. Increased late mortality after sirolimus-eluting stents versus bare-metal stents in diseased saphenous vein grafts: results from the randomized DELAYED RRISC Trial. J Am Coll Cardiol. 2007; 50(3):261-7.

11. Assali A, Raz Y, Vaknin-Assa H, Ben-Dor I, Brosh D, Teplitsky I, et al. Beneficial 2-years results of drug-eluting stents in saphenous vein graft lesions. Eurolntervention. 2008;4(1): 108-14.

12. Vignali L, Saia F, Manari A, Santarelli A, Rubboli A, Varani $\mathrm{E}$, et al. Long-term outcomes with drug-eluting stents versus bare metal stents in the treatment of saphenous vein graft disease (results from the REgistro Regionale AngiopLastiche Emilia-Romagna registry). Am J Cardiol. 2008;101(7):947-52.

13. Ge L, lakovou I, Sangiorgi GM, Chieffo A, Melzi G, Cosgrave J, et al. Treatment of saphenous vein graft lesions with drugeluting stents: immediate and midterm outcome. J Am Coll Cardiol. 2005;45(7):989-94.

14. Chu WW, Kuchulakanti PK, Wang B, Clavijo LC, Suddath WO, Pichard AD, et al. Efficacy of sirolimus-eluting stents as compared to paclitaxel-eluting stents for saphenous vein graft intervention. J Interv Cardiol. 2006;19(2):121-5.

15. Lee MS, Shah AP, Aragon J, Jamali A, Dohad S, Kar S, et al. Drugeluting stenting is superior to bare metal stenting in saphenous vein grafts. Catheter Cardiovasc Interv. 2005;66(4):507-11.

16. Brodie BR, Wilson $H$, Stuckey $T$, Nussbaum $M$, Laurent $S$, Bradshaw B, et al. STENT Group. Outcomes with drugeluting versus bare-metal stents in saphenous vein graft intervention results from the STENT (Strategic Transcatheter 
Evaluation of New Therapies) group. JACC Cardiovasc Interv. 2009;2(11):1105-12.

17. van Twisk PH, Daemen J, Kukreja N, van Domburg RT, Serruys PW. Four-year safety and efficacy of the unrestricted use of sirolimus- and paclitaxel-eluting stents in coronary artery bypass grafts. Eurolntervention. 2008;4(3):311-7.

18. Okabe T, Lindsay J, Buch AN, Steinberg DH, Roy P, Slottow $\mathrm{TL}$, et al. Drug-eluting stents versus bare metal stents for narrowing in saphenous vein grafts. Am J Cardiol. 2008; 102(5):530-4.

19. Applegate RJ, Sacrinty M, Kutcher M, Santos R, Gandhi S, Little W. Late outcomes of drug-eluting versus bare metal stents in saphenous vein grafts: Propensity score analysis. Catheter Cardiovasc Interv. 2008;72(1):7-12.

20. Goswami NJ, Gaffigan M, Berrio G, Plessa AL, Pfeiffer AM, Markwell SJ, et al. Long-term outcomes of drug-eluting stents versus bare-metal stents in saphenous vein graft disease: results from the Prairie "Real World" Stent Registry. Catheter Cardiovasc Interv. 2010;75(1):93-100.

21. Meier P, Brilakis ES, Corti R, Knapp G, Shishehbor MH, Gurm HS. Drug-eluting versus bare-metal stent for treatment of saphenous vein grafts: a meta-analysis. PLoS One. 2010; 5(6):e11040.

22. Sanchez-Recalde A, Jiménez Valero S, Moreno R, Barreales L, Lozano I, Galeote G, et al. Safety and efficacy of drug-eluting stents versus bare-metal stents in saphenous vein grafts lesions: a meta-analysis. Eurolntervention. 2010;6(1):149-60.

23. Rodés-Cabau J, Bertrand OF, Larose E, Déry JP, Rinfret S, Bagur R, et al. Comparison of plaque sealing with paclitaxeleluting stents versus medical therapy for the treatment of moderate nonsignificant saphenous vein graft lesions: the moderate vein graft lesion stenting with the Taxus stent and intravascular ultrasound (VELETI) pilot trial. Circulation. 2009; 120(20):1978-86. 